\title{
Proposta de instrumento para projeto terapêutico singular em saúde mental
}

\author{
Proposal for an instrument for a unique therapeutic project in mental \\ health
}

\author{
1 Jorcilene Alcântara Silva Laurito \\ 1 Vagner Ferreira do Nascimento vagnerschon@hotmail.com \\ 2 Alisséia Guimarães Lemes
}

1 Universidade do Estado de Mato Grosso (UNEMAT)
2 Universidade Federal de Mato Grosso (UFMT)

\section{Resumo}

O estudo teve por objetivo apresentar proposta de instrumento para a execução de projeto terapêutico singular, no atendimento às demandas de saúde mental na Atenção Básica. Trata-se de uma pesquisa-ação realizada entre junho e setembro de 2017, em um município do médio norte de Mato Grosso. A primeira versão do instrumento foi encaminhada para apreciação de enfermeiros de unidades de saúde da família do município do estudo. Ao término dessa etapa, o instrumento gerado foi submetido a teste piloto com duração de 30 dias, junto a uma usuária do serviço de saúde, com diagnóstico de doença mental. Após essa etapa, verificou-se que o instrumento demonstrou fácil aplicabilidade, porém as dificuldades encontradas voltaram-se à dinâmica e organização do serviço de saúde em realizar visitas domiciliares com maior frequência, a fim de avaliar a efetividade da intervenção terapêutica. Apesar de existir certa resistência à adesão dessa estratégia, acredita-se que esse instrumento poderá auxiliar o trabalho dos profissionais desse setor na gestão do cuidado, bem como possibilitar atendimento integral e baseado nas necessidades do cliente e família. Além disso, espera-se que o instrumento sirva de suporte ou direcionamento para a concretização do projeto terapêutico singular.

\section{Palavras-chave}

Saúde pública. Assistência integral à saúde. Saúde mental.

\begin{abstract}
The purpose of this study was to present a proposal for an instrument for the execution of a unique therapeutic project in the care of mental health demands in Primary Care. It is an action research carried out between June and September of 2017 in a municipality in the middle north of Mato Grosso. The first version of the instrument was sent to nurses at the health units of the study municipality. At the end of that stage, the generated instrument was submitted to a 30day pilot test with a health care user diagnosed with mental illness. After this step, it was verified that the instrument demonstrated easy applicability, but the difficulties encountered went back to the dynamics and organization of the health service in making home visits more frequently in order to evaluate the effectiveness of the therapeutic intervention. Although there is some resistance to adherence to this strategy, it is believed that this instrument may assist the work of professionals in this sector in the management of care, as well as provide comprehensive care based on the needs of the client and family. In addition, it is expected that the instrument will serve as support or guidance for the implementation of the unique therapeutic project.
\end{abstract}

\section{Keywords}

Public health. Comprehensive health care. Mental health.

\section{Como você deve citar?}

LAURITO, Jorcilene Alcântara Silva; NASCIMENTO, Vagner Ferreira do; LEMES, Alisséia Guimarães. Proposta de instrumento para projeto terapêutico singular em saúde mental. Cadernos UniFOA, Volta Redonda, n. 37, p. 115122, ago. 2018. 


\section{INTRODUÇÃO}

No Brasil, a atual política de saúde mental é resultado da mobilização de usuários, famílias e profissionais de saúde, quando, por volta dos anos 80 , através dos movimentos de lutas Antimanicomiais e da Reforma psiquiátrica, objetivaram mudar a realidade da assistência às pessoas com transtornos mentais e pôr fim aos manicômios. Em 2001, após um longo período de tramitação no Congresso nacional, foi sancionada a lei $n^{0} 10.2016$, que garante os direitos das pessoas com transtornos mentais e redireciona o modelo assistencial em saúde mental. Posteriormente, o decreto presidencial $n{ }^{\circ}$ $7508 / 2011$ ampliou as redes de atenção psicossocial (RAPS), através da criação de serviços substitutivos ao sistema manicomial (BRASIL, 2013).

Nesse cenário de mudanças, também surgiu a Estratégia de Saúde da Família (ESF), afırmando-se como um importante aliado nessa rede, pois contribui com a integralidade e efetividade da assistência. Um dos maiores desafios no contexto da ESF foi a integração entre a saúde mental e a saúde da família, o que traduziu um ponto de partida na busca de resoluções e cuidados em saúde mental (WETZEL et al., 2014).

A humanização e a integralidade do cuidado são alvo a serem alcançados pelos trabalhadores e serviços de saúde. E, para que isso aconteça, é necessário repensar as estratégias de ações, a fim de colocar o usuário como sujeito ativo na produção do cuidado voltado à sua saúde. Um exemplo de estratégia que pode ser implementada é o Projeto Terapêutico Singular (PTS) (SILVA et al., 2013).

O PTS é um dispositivo de intervenção que possibilita a organização do processo de trabalho da equipe de saúde, favorecendo o atendimento integral aos casos que demandam maior gravidade e complexidade (BRASIL, 2013). O PTS é considerado uma forte ferramenta na gestão do cuidado, com possibilidade de alcançar resultados expressivos, por meio de acolhimento qualificado, estabelecimento de vínculo, divisão de tarefas e responsabilização compartilhada entre a equipe de saúde e usuários (DORIGATTI et al., 2014).

Com base nas afirmações dos autores supracitados, a introdução do PTS como prática de cuidados em saúde mental na Atenção Básica é justificada pela importância da estruturação da rede de atenção psicossocial, na defesa pelos ideais estabelecidos a partir da Reforma Psiquiátrica brasileira e na valorização da vida de indivíduos e famílias em sofrimento psíquico.

Diante disso, o objetivo deste estudo foi apresentar proposta de instrumento para a execução de projeto terapêutico singular no atendimento às demandas de saúde mental na Atenção Básica.

\section{MÉTODOS}

Trata-se de uma pesquisa-ação realizada entre junho e setembro de 2017, em um município do médio norte de Mato Grosso, fundamentado na experiência de Hahn (2015), que retrata de pesquisa para o desenvolvimento de novas metodologias, processos e instrumentos, pois se baseia no conhecimento do problema e pessoas para quais serão direcionadas a intervenção, com estruturação em um planejamento cuidadoso entre uma equipe diversificada que, por fim, desenvolva uma teoria de intervenção que oriente a investigação.

Seguiu-se também a definição de que o PTS é um conjunto articulado de ações a serem efetivadas no cotidiano do serviço, pautadas em quatro movimentos para o desenvolvimento do material: 
1 - diagnóstico situacional, 2 - definição de metas, 3- divisão de responsabilidades e 4- reavaliação (BRASIL, 2013).

A primeira versão do instrumento criado foi desenvolvida pelos próprios pesquisadores do estudo, levando em consideração a vasta experiência de ambos na Atenção Básica e no cuidado de pessoas em sofrimento psíquico.

Esse instrumento foi encaminhado para todos (23) enfermeiros das unidades de saúde da família (USF) do município investigado, juntamente com o Termo de Consentimento Livre e Esclarecido (TCLE) e uma carta de apresentação do estudo e convite para realizarem apreciação da primeira versão do instrumento, em relação à clareza, importância das informações e estrutura apresentada. Para isso, os pesquisadores forneceram um roteiro de observação com questões objetivas para o preenchimento. Somente 18 enfermeiros deram a devolutiva do instrumento. Após essa etapa, partiu-se para a construção final do instrumento, atendendo às indicações desses profissionais.

De forma complementar, o estudo também propôs um teste piloto com o instrumento gerado. Assim, de forma aleatória, escolheu-se uma das equipes de USF do município, sorteando também uma usuária com demandas em saúde mental para ser assistida com o instrumento. 0 feedback dessa equipe com os pesquisadores ocorreu através dos relatórios das visitas domiciliares e diários de campo.

O estudo respeitou todos aspectos éticos em pesquisa, conforme a resolução 466/2012, iniciando-se somente após a aprovação do Comitê de Ética em Pesquisa com Seres Humanos (CEP), da Universidade Federal de Mato Grosso (UFMT), sob o protocolo número 515/705 e CAAE: 18394713.0.0000.5587.

\section{RESULTADOS E DISCUSSÃO}

O PTS foi construído com o intuito de contribuir com a prática profissional no atendimento às demandas em saúde, em especial demandas em saúde mental.

A versão final do modelo de PTS contempla as seguintes etapas: identificação do paciente (dados pessoais, micro área, genograma e ecomapa, hipótese diagnóstica, medicações em uso, acompanhamento em outro setor e os principais problemas/ agravos identificados). 0 próximo item é composto pelas metas, plano terapêutico e planejamento das atividades, seguido pelos responsáveis pelo seguimento do plano terapêutico; e, por último, a reavaliação do usuário, evolução do caso, mudanças ou manutenção do plano terapêutico e agendamento da próxima reavaliação (Apêndice A).

Para execução do teste piloto, foi escolhida uma usuária do Sistema Único de Saúde (SUS) com conhecida história de demanda em saúde mental. Após a escolha, realizou-se visita domiciliar, a fim de se apresentar a nova proposta de acompanhamento terapêutico, com explicação breve e objetiva do PTS. As etapas que compreenderam o diagnóstico situacional, definição de metas, plano terapêutico e divisão de responsabilidades foram realizadas e definidas em comum acordo entre a equipe multiprofissional, a usuária e seu companheiro. Para a etapa de reavaliação mensal, foi estabelecido o prazo de 30 dias.

Até essa fase de execução do PTS, observou-se que o instrumento demonstrou fácil aplicabilidade. Porém, o maior desafio encontrado foi a organização em se estabelecer cronograma de visitas periódicas e disponibilidade de período/agenda da equipe para a discussão do caso.

Em relação ao feedback realizado pelos enfermeiros que receberam o instrumento do PTS para a apreciação, verificou-se que somente um profissional referiu utilizar os conhecimentos do PTS para 
assistir os usuários em sofrimento psíquico, porém sem auxílio de instrumento norteador. E, mesmo sem apropriação de algum instrumento de PTS em seus locais de trabalho, esses profissionais expressaram a importância da disponibilidade dessa ferramenta proposta pelo estudo.

Essa realidade também já foi apontada por outros pesquisadores, os quais revelam que, embora o PTS não seja uma prática regular em todas as equipes de saúde, alguns profissionais utilizam a lógica desse instrumento em suas práticas de cuidado. No entanto, para que o PTS se torne uma estratégia de cuidado eficaz, faz-se necessário a criação de um modelo estruturado que favoreça o cuidado terapêutico (SILVA et al., 2016).

A avaliação global do instrumento foi positiva para todos os profissionais, destacando-se, principalmente, a praticidade, facilidade de aplicação, possibilidade de organização da assistência e resolutividade das ações do território. Entretanto, como desvantagens, manifestaram dificuldades no processo de referência e contrarreferência em saúde mental do município; relataram que esse instrumento seria uma "tarefa" a mais - considerando que já existe uma grande quantidade de outros relatórios a serem executados mensalmente; e citaram uma possível dificuldade de adesão dos profissionais, usuários e famílias.

Com base nesses achados, alguns autores também já observaram a dificuldade de adesão ao instrumento como possível desvantagem, pois consideram que a adesão terapêutica se constitui como um processo operacional complexo que necessita identificar os fatores interferentes para assim propor estratégias para o seu enfrentamento (VIDAL et al., 2017). Paralelamente, outros pesquisadores também relacionaram que a operacionalização do PTS nas equipes que ainda não incorporaram essa ferramenta pode gerar tensões e divergências (HORI; NASCIMENTO, 2014).

Conforme estudo de Jorge et al. (2015), algumas estratégias devem ser desenvolvidas, a fim de favorecerem a utilização de práticas inovadoras no campo de saúde mental, sendo elas, a implementação de processos de educação permanente, estímulo ao trabalho interdisciplinar e flexibilização de cronograma que favoreçam o acolhimento e condução de casos de saúde mental e melhor estruturação dos centros de saúde.

Neste estudo, o entendimento demonstrado pelos profissionais que apreciaram o instrumento aponta a importância e a complexidade da inclusão do PTS no atendimento às demandas de saúde. Desse modo, o desenvolvimento do PTS se constitui como possibilidade de mudança e fortalecimento da gestão do cuidado na ESF, permitindo que o cuidado seja adequado às necessidades do indivíduo e família (PEIXOTO; CARVALHO; VILASBOAS, 2017).

De acordo com Grigolo et al. (2015), o PTS é uma estratégia terapêutica de cuidado já usada em vários contextos e pontos de atenção das redes e pode ser ampliada para a Atenção Básica. 0 autor ressalta também que trabalhar com o PTS é colocar-se à disposição para assumir riscos, permitir encontros de diferenças, manter-se em andamento, com definições, soluções e aprofundamentos.

\section{CONSIDERAÇÕES FINAIS}

A experiência em elaborar e executar do PTS permitiu aprofundar as possibilidades de atendimentos aos usuários com demandas em saúde mental, especialmente no contexto da atenção básica. Acredita-se que o instrumento norteador proposto possa auxiliar o trabalho dos profissionais da ESF na gestão do cuidado, bem como propiciar atendimento integral e baseado nas necessidades do cliente e família. Além disso, espera-se que o instrumento sirva de suporte ou direcionamento para a efetivação 
do PTS em outras realidades em saúde. Todavia, mesmo conseguindo estruturar instrumento para a aplicação do PTS, há necessidade de novos estudos de modo sistemático, em que ocorra a implantação e observação desse instrumento na rotina dos serviços de saúde com possíveis impactos na qualidade da assistência oferecida.

\section{REFERÊNCIAS}

BRASIL. Ministério da Saúde. Cadernos de Atenção Básica - Saúde Mental. Departamento de Atenção Básica. Brasília: Ministério da Saúde, 2013.

DORIGATTI, A. E. et al. Projeto Terapêutico Singular no âmbito da Saúde Mental: Uma experiência no curso de graduação em Medicina. Rev. bras. educ. med., v. 38, n. 1, p. 113-119, 2014.

GRIGOLO, T. M. et al. O Projeto terapêutico Singular na Clínica da Atenção Psicossocial. Cad. bras. saúde mental, v. 7, n. 15, p.53-73, 2015.

HANH, G. V. Incluindo o Projeto Terapêutico Singular na Agenda da Atenção Básica em contexto de vulnerabilidade e não adesão ao tratamento de tuberculose. 2015. $207 \mathrm{f}$. Tese (Doutorado em Enfermagem) - Escola de Enfermagem, Universidade Federal do Rio Grande do Sul, Porto Alegre, 2015.

HORI, A. A.; NASCIMENTO, A. F. O Projeto Terapêutico Singular e as práticas de saúde mental nos Núcleos de Apoio à Saúde da Família (NASF) em Guarulhos (SP), Brasil. Ciênc. saúde colet., v. 19, n. 8 , p. 3561-3571, 2014.

JORGE, M. S. B. et al. Apoio Matricial, Projeto Terapêutico Singular e produção do cuidado em Saúde Mental. Texto Contexto Enferm., v. 24, n.1, p. 112-20, 2015.

PEIXOTO, M. T.; CARVALHO, R. C.; VILASBOAS, A. L. Q. Projeto Terapêutico Familiar: Uma experiência da Atenção Integral em uma Unidade de Saúde da Família num município do semiárido Baiano. Rev. saúde col. UEFS, v. 7, n. 2, p. 35-43, 2017.

SILVA, A. I. et al. Projeto Terapêutico Singular para profissionais da Estratégia de Saúde da Família. Cogitare enferm., v. 21, n. 3, p. 1-8, 2016.

SILVA, E. P.; MELO, F. A. B. P.; SOUSA, M. M. Projeto terapêutico Singular como estratégia de prática da multiprofissionalidade nas ações de saúde. Rev. bras. ciênc. saúde, v. 17, n. 2, p. 197-202, 2013.

VIDAL, L. M. S. et al. Projeto Terapêutico Familiar: Uma experiência da Atenção Integral em uma Unidade de Saúde da Família num município do semiárido Baiano. Rev. Saúde Col. UEFS, v. 7, n. 2, p. 59-64, 2017.

WETZEL, C. et al. A rede de atenção à saúde mental a partir da Estratégia Saúde da Família. Rev. gaúch. enferm, v. 35, n. 2, p. 27-32, 2014. 
APÊNDICE A

INSTRUMENTO - PROJETO TERAPÊUTICO SINGULAR (PTS)

\section{Identificação}

a. Nome do paciente:

b. DN: idade:

c. Micro área: $\mathrm{n}^{\circ}$ prontuário:

d. Endereço: Telefone: ( )

e. Genograma e Ecomapa Data da elaboração:_/_/_

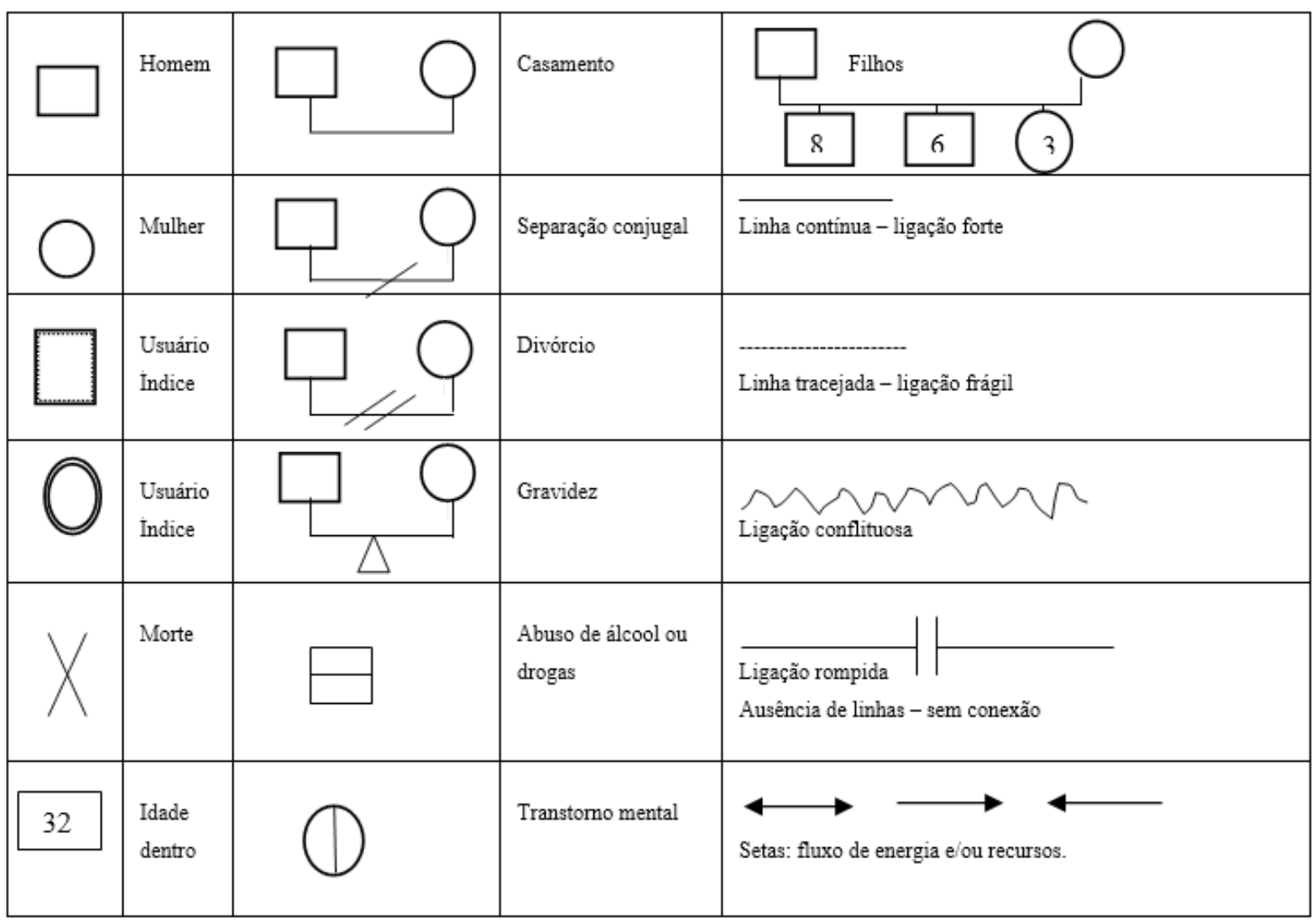

Fonte: Caderno de atenção domiciliar (2013). 
f. Hipótese diagnóstica:

g. Medicação em uso:

h. Está sendo acompanhado por outro setor? ( ) Sim ( ) Não. Qual?

i. Principais problemas/ agravos identificados:

2. Metas

- Possibilitar a participação do usuário e da família no cuidado.

- Garantir tratamento digno e de qualidade ao usuário em sofrimento mental.

3. Plano terapêutico:
( ) Atendimento individual
) Participação em grupos da ESF/ QUAIS?
) Participação na igreja ou grupos religiosos
( ) Atividade física / ONDE:
( ) Atividades culturais / QUAIS:
( ) Práticas integrativas complementares / QUAIS:
( )
( ) )

Quadro 1 - Planejamento semanal (definido com o usuário e/ou família)

\begin{tabular}{|c|c|c|c|c|c|c|c|}
\hline & Segunda & Terça & Quarta & Quinta & Sexta & Sábado & Domingo \\
\hline Manh & & & & & & & \\
\hline Tarde & & & & & & & \\
\hline Noite & & & & & & & \\
\hline
\end{tabular}

4. Responsáveis

( ) Usuário ( ) Familiares ( ) Cuidador (não-familiar)

( ) Médico ( ) Enfermeiro ( ) ACS ( ) Dentista

( ) Outros membros da equipe

5. Reavaliação mensal

a. Evolução do caso: 
Proposta de instrumento para projeto terapêutico singular em saúde mental

b. Avaliações do Plano Terapêutico:

(R) Resolvido (M) Mantido (MP) Mudança de plano terapêutico

c. Próxima reavaliação:

Assinatura do paciente:

Assinatura do familiar responsável:

Assinatura do(s) profissional (is): 